\title{
Antireflux Metal Stent for Initial Treatment of Malignant Distal Biliary Obstruction
}

\author{
Shinichi Morita ${ }^{D},{ }^{1}$ Yasuaki Arai, ${ }^{2}$ Shunsuke Sugawara, ${ }^{2}$ Miyuki Sone, ${ }^{2}$ Yasunari Sakamoto, ${ }^{3}$ \\ Takuji Okusaka, ${ }^{3}$ Shigetaka Yoshinaga, ${ }^{4}$ Yutaka Saito $\left(\mathbb{D}^{4},{ }^{4}\right.$ and Shuji Terai ${ }^{5}$ \\ ${ }^{1}$ Department of Gastroenterology and Hepatology, Uonuma Institute of Community Medicine, Niigata University Hospital, \\ 4132 Urasa, Minamiuonuma, Niigata 949-7302, Japan \\ ${ }^{2}$ Department of Diagnostic Radiology, National Cancer Center Hospital, 5-1-1 Tsukiji, Chuo-ku, Tokyo 104-0045, Japan \\ ${ }^{3}$ Department of Hepatology and Pancreatobiliary Internal Medicine, National Cancer Center Hospital, 5-1-1 Tsukiji, Chuo-ku, \\ Tokyo 104-0045, Japan \\ ${ }^{4}$ Endoscopy Division, National Cancer Center Hospital, 5-1-1 Tsukiji, Chuo-ku, Tokyo 104-0045, Japan \\ ${ }^{5}$ Department of Gastroenterology and Hepatology, Niigata University Hospital, 754 Ichibancho, Asahimachidori, Chuo-ku, \\ Niigata city, Niigata 951-8510, Japan
}

Correspondence should be addressed to Shinichi Morita; m0riz0u@extra.ocn.ne.jp

Received 1 October 2017; Accepted 2 January 2018; Published 31 January 2018

Academic Editor: Niccola Funel

Copyright (c) 2018 Shinichi Morita et al. This is an open access article distributed under the Creative Commons Attribution License, which permits unrestricted use, distribution, and reproduction in any medium, provided the original work is properly cited.

Objectives. To compare the use of an antireflux metal stent (ARMS) with that of a conventional covered self-expandable metal stent (c-CSEMS) for initial stenting of malignant distal biliary obstruction (MDBO). Materials and Methods. We retrospectively investigated 59 consecutive patients with unresectable MDBO undergoing initial endoscopic biliary drainage. ARMS was used in 32 patients and c-CSEMS in 27. Technical success, functional success, complications, causes of recurrent biliary obstruction (RBO), time to RBO (TRBO), and reintervention were compared between the groups. Results. Stent placement was technically successful in all patients. There were no significant intergroup differences in functional success (ARMS [96.9\%] versus c-CSEMS [96.2\%]), complications (6.2 versus 7.4\%), and RBO (48.4 versus 42.3\%). Food impaction was significantly less frequent for ARMS than for c-CSEMS $(P=0.037)$, but TRBO did not differ significantly between the groups (log-rank test, $P=0.967$ ). The median TRBO was 180.0 [interquartile range (IQR), 114.0-349.0] days for ARMS and 137.0 [IQR, 87.0-442.0] days for c-CSEMS. In both groups, reintervention for RBO was successfully completed in all patients thus treated. Conclusion. ARMS offers no advantage for initial stent placement, but food impaction is significantly prevented by the antireflux valve.

\section{Introduction}

For patients with unresectable malignant distal biliary obstruction (MDBO), endoscopic placement of a selfexpandable metal stent (SEMS) is a widely accepted treatment for the relief of jaundice [1-4]. SEMS has been used not only as palliative therapy but also prior to chemotherapy. The survival time for patients with pancreatic cancer has been improving, due mainly in part to recent advances in chemotherapeutic intervention [5-7].
Therefore, prolongation of SEMS patency is desirable to allow continuation of anticancer treatment and thus improve patient prognosis.

SEMS remains patent for longer than a plastic stent because of its wide lumen $[8,9]$. The diameter of the SEMS is generally more than $8 \mathrm{~mm}$ after placement, allowing a relative smoothness and reduction of sludge accumulation that can compromise stent function. However, SEMS dysfunction does occur occasionally after placement, leading to cholangitis that requires reintervention. 
In cases of MDBO, the SEMS is usually placed across the papilla. However, this is reported to increase the risk of occlusion due to loss of sphincter function [10], and consequent duodenobiliary reflux can lead to sludge accumulation [11]. To prevent this and to prolong stent patency, the antireflux metal stent (ARMS) was developed, and many studies have reported that this is more effective than ordinary stents [12-18]. However, as reported by Ustundag et al., the superiority of the ARMS over the ordinary covered SEMS has not been verified, especially for initial stenting [19], and therefore the effectiveness of ARMS for MDBO remains controversial.

In the present study, we retrospectively compared the effectiveness of the ARMS versus the conventional covered SEMS (c-CSEMS) for initial stenting in patients with unresectable MDBO.

\section{Materials and Methods}

This study was approved by the institutional Human Investigation Committee of the National Cancer Center Japan (2014-322), and written informed consent was obtained from all patients in accordance with the tenets of the Declaration of Helsinki.

2.1. Patients. Between February 2013 and December 2014, 59 consecutive patients ( 31 men and 28 women; median age, 67 years; range, 23-88 years) with unresectable MDBO were treated with SEMS and enrolled in this study. Patients with hilar biliary obstruction and surgically altered gastrointestinal anatomy, or those with a performance status lower than 4 according to the Eastern Cooperative Oncology Group [20] scale, were excluded. The SEMS was placed endoscopically in all patients. c-CSEMS placement was performed in 27 consecutive patients (13 men and 14 women; median age, 65 years; range, 23-88 years; c-CSEMS group) between February 2013 and March 2014, and ARMS placement was performed in 32 consecutive patients (18 men and 14 women; median age, 71 years; range, 43-87 years; ARMS group) between March and December 2014. The underlying diseases were pancreatic cancer in 50 patients, bile duct cancer in 4, lymph node metastases in 4, and ampullary cancer in 1 . The diagnosis was based on imaging modalities such as computed tomography, magnetic resonance imaging, and endoscopic retrograde cholangiopancreatography (ERCP). Malignancy was confirmed pathologically by endoscopic ultrasound-guided fine-needle aspiration, bile duct biopsy, or brushing cytology. All patients were followed up from the time of stent placement to the recurrent biliary obstruction $(\mathrm{RBO})$ or death if the patients were not obstructed by their stents.

2.2. Stent Designs. The characteristics of the inserted stents are shown in Table S1. The ARMS [13] (Niti-S long covered ComVi stent, Taewoong Medical Inc., Gimpo, Korea) is manufactured on the basis of the Niti-S ComVi SEMS [21] and has a funnel-shaped antireflux valve attached at its distal end (Figure S1 A and B). The valve portion of the stent is $7 \mathrm{~mm}$ long. The inner and outer layers of this stent are braided with nitinol wire, and an e-polytetrafluoroethylene
(e-PTFE) membrane is sandwiched between these layers. The axial force of the inner and outer layers is weak, and the membrane is not fixed to the wire mesh. Thus, the axial force of the ComVi stent is weak, and the membrane fully covers the stent. The ARMS employed was $10 \mathrm{~mm}$ in diameter, and the metallic portion was available in lengths of $60 \mathrm{~mm}$ and $80 \mathrm{~mm}$.

The c-CSEMS used in this study was the Niti-S SUPREMO stent (Taewoong Medical Inc., Gimpo, Korea) [22], which is braided with nitinol and has characteristic irregularly knitted stent cells (Figure S1 C). The large and small cells alter the amount of radial force, which is able to increase and decrease to allow the stent to fit into the bile duct. The stent membrane is made of silicone integrated with wire mesh, fully covering the stent. It was $10 \mathrm{~mm}$ in diameter and was available in lengths of $60 \mathrm{~mm}$ and $80 \mathrm{~mm}$. Both ends of this stent were slightly flared.

2.3. Procedures. All SEMSs were placed using an ERCP technique. All of the patients underwent ERCP with a standard side-viewing duodenoscope (JF260V or TJF260V; Olympus Optical Co., Tokyo, Japan). All procedures were performed under conscious sedation, and all patients received prophylactic antibiotics. Sphincterotomy was performed before stent insertion in all cases. After evaluation of biliary stricture by cholangiography, a 0.025 -inch guidewire (Visiglide or Visiglide 2; Olympus Optical Co., Tokyo, Japan) was passed through the stricture and inserted into the hepatic bile duct. The c-CSEMS or ARMS delivery system was inserted into the bile duct over the prepositioned guidewire, and the stent was deployed under fluoroscopic and endoscopic guidance. In all cases, the stent was placed across the papilla and the metallic portion extended into the duodenum for approximately one centimeter. The length of the stent was determined on the basis of cholangiographic findings.

All patients were followed up clinically, and complete blood counts and liver function tests were performed within one week after stenting, being repeated every month thereafter.

If symptoms of RBO such as high fever, upper abdominal pain, and/or jaundice occurred, reintervention was performed if an endoscopic procedure was permissible. For cleaning of the lumina of problematic stents with modest sludge accumulation, a balloon catheter for stone removal was employed. On the other hand, stents that had become almost or completely occluded were grasped with alligator forceps and removed carefully. In cases where the stent had migrated proximally (i.e., into the common bile duct), the stent was similarly and carefully pulled up and out through the duodenum. A new metal stent was then deployed, and the replacement stent was the same type as the previous one.

2.4. Study Design. This study was designed as a singleinstitution retrospective study. Evaluation of stent treatment was based on the TOKYO criteria 2014, which is a standardized system for reporting the status of biliary stents [23]. The primary outcome of this study was the time to RBO (TRBO). $\mathrm{RBO}$ was defined as the recurrence of obstructive jaundice and/or cholangitis due to stent occlusion or migration. TRBO was defined as the length of time between stent placement 
and the point of RBO. The secondary outcomes included technical success, functional success, complications, causes of RBO, risk factors for RBO, and reintervention. Technical success was defined as successful deployment of a stent with sufficient coverage of the stricture. Functional success was defined as a $50 \%$ decrease in or normalization of the bilirubin level to a standard value used at our institution within 14 days of stent placement.

Causes of stent occlusion such as sludge accumulation and food impaction were determined when reintervention revealed a large amount of sludge or food residue, respectively, in an occluded SEMS. Stent migration was diagnosed when a reintervention revealed a completely or partially migrated SEMS as a cause of RBO.

2.5. Statistical Analysis. Statistical analyses were performed using SPSS software for Windows version 22.0 (SPSS Inc., Chicago, IL). Differences between groups were compared using the Mann-Whitney $U$ test, and differences in proportions were compared using Fisher's exact test. KaplanMeier estimation of TRBO was performed, and survival curves were compared by the log-rank test. Patient death was treated as censored at the time of death. Univariate and multivariate analyses were performed to identify risk factors for RBO using the Cox proportional hazards model. The model included age ( $<70$ versus $\geqq 70$ years), sex, stent type (ARMS versus c-CSEMS), tumor etiology, complicating cholangitis, tumor invasion of the duodenum, the level of serum total bilirubin $(<3.0$ versus $\geqq 3.0 \mathrm{mg} / \mathrm{dL})$, use of oral ursodeoxycholic acid after stenting, and adjuvant chemotherapy. Statistical significance was defined as $P<0.05$. The statistical methods used in this study were reviewed by Stagen Co. Ltd.

\section{Results}

3.1. Patient Characteristics. Patient characteristics and clinical details of the ARMS and c-CSEMS groups are summarized in Table 1. There were no significant intergroup differences in sex, age, diagnosis, duodenal invasion, chemotherapy, or oral ursodeoxycholic acid use after stenting.

3.2. Outcomes. Technical success was achieved in all patients (100\%). Functional success was achieved in 31 of the 32 patients (96.9\%) in the ARMS group and 26 of the 27 patients (96.2\%) in the c-CSEMS group. The median procedure time did not differ significantly between the groups.

Procedure-related complications occurred in two patients (6.2\%) in the ARMS group and two (7.4\%) in the c-CSEMS group; the intergroup difference was not significant. Moderate cholecystitis developed in one patient in the ARMS group and two in the c-CSEMS group. These patients underwent percutaneous transhepatic gallbladder drainage and were administered prophylactic antibiotics. Their condition improved in a short time without cholecystectomy. In the ARMS group, one patient suffered liver failure due to portal vein occlusion because of the pressure of stent expansion and the jaundice did not improve. There was no procedure-related mortality.
TABLE 1: Patients' characteristics and clinical details of the ARMS and c-CSEMS groups.

\begin{tabular}{lccc}
\hline & $\begin{array}{c}\text { ARMS } \\
\text { group }\end{array}$ & $\begin{array}{c}\text { c-CSEMS } \\
\text { group }\end{array}$ & P value \\
\hline Number of patients & 32 & 27 & \\
Gender (male/female) & $18 / 14$ & $13 / 14$ & 0.606 \\
Median age (range) (years) & $71(43-87)$ & $65(23-88)$ & 0.825 \\
Diagnosis & & & 0.901 \\
$\quad$ Pancreatic cancer & 28 & 22 & \\
$\quad$ Bile duct cancer & 3 & 1 & \\
$\quad$ Ampullary cancer & 0 & 1 & \\
$\quad$ Lymph node metastases & 1 & 3 & \\
Duodenal invasion (\%) & $7(21.9)$ & $9(33.3)$ & 0.386 \\
Chemotherapy (\%) & $22(68.8)$ & $17(63.0)$ & 0.640 \\
Oral ursodeoxycholic acid (\%) & $7(21.9)$ & $9(33.3)$ & 0.386 \\
\hline
\end{tabular}

ARMS: antireflux metal stent; c-CSEMS: conventional covered selfexpandable metal stent.

RBO occurred in 15 patients (48.4\%) in the ARMS group and $12(46.2 \%)$ in the c-CSEMS group. In the ARMS group, the causes of RBO were tumor overgrowth in 1 patient, sludge accumulation (Figure S2 A and B) in 9, and symptomatic migration in 5. Migration was distal in 3 patients and proximal in 2 (Figure S2 C). In the c-SEMS group, RBO was due to tumor overgrowth in 1 patient, sludge accumulation in 5 , food impaction in 4 , and distal migration in 2 . The incidence of food impaction was significantly higher for cCSEMS than for ARMS $(P=0.037)$. Outcomes of ARMS are shown in Table 2 .

3.3. Time to Recurrent Biliary Obstruction. Kaplan-Meier estimation of TRBO was performed for patients achieving functional success. The results of the log-rank test showed no significant intergroup difference in TRBO (log-rank, $P=$ 0.967 ; Figure 1). Rates of nonobstruction at 3, 6, and 12 months were $82 \%, 50 \%$, and $19 \%$, respectively, for ARMS and $72 \%, 48 \%$, and $32 \%$, respectively, for c-CSEMS. The median TRBO was 180.0 [interquartile range (IQR), 114.0 to 349.0] days for ARMS and 137.0 [IQR, 87.0 to 442.0] days for c-CSEMS.

3.4. Risk Factors for Recurrent Biliary Obstruction. The results of univariate and multivariate analyses for identifying RBO risk factors are shown in Table S2. There were no factors significantly predictive of $\mathrm{RBO}$, including the stent type.

3.5. Reintervention. In the ARMS group, 14 patients underwent reintervention at the time of RBO. One patient improved after antibiotic administration alone. Two patients with modest sludge accumulation in the stent underwent cleaning of the stent lumen with a balloon catheter for stone removal and drainage with a nasal biliary drainage tube. Seven patients underwent removal of the occluded stent with alligator forceps and replacement with a new ARMS. Two patients with RBO due to proximal migration underwent careful removal of the stent from the common bile duct using alligator forceps without any problems and then received a 
TABLE 2: Clinical outcomes of the ARMS and c-CSEMS groups.

\begin{tabular}{|c|c|c|c|}
\hline & ARMS group $(n=32)$ & c-CSEMS group $(n=27)$ & $P$ value \\
\hline Technical success (\%) & $32 / 32(100)$ & $27 / 27(100)$ & - \\
\hline Functional success (\%) & $31 / 32(96.9)$ & $26 / 27(96.2)$ & 1.000 \\
\hline Median procedure time (min) & $30(15-50)$ & $40(20-70)$ & 0.735 \\
\hline Complications (\%) & $2(6.2)$ & $2(7.4)$ & 1.000 \\
\hline Cholecystitis & 1 & 2 & 0.588 \\
\hline Liver failure & 1 & 0 & 1.000 \\
\hline Recurrent biliary obstruction (\%) & $15 / 31(48.4)$ & $12 / 26(46.2)$ & 0.866 \\
\hline Overgrowth & 1 & 1 & 1.000 \\
\hline Sludge accumulation & 9 & 5 & 0.584 \\
\hline \multirow[t]{2}{*}{ Food impaction } & 0 & 4 & $0.037^{*}$ \\
\hline & 5 & 2 & \\
\hline \multirow[t]{2}{*}{ Migration } & Distal 3 & Distal 2 & 0.436 \\
\hline & Proximal 2 & Proximal 0 & \\
\hline Reintervention (\%) & $14 / 15(93.3)$ & $11 / 12(91.7)$ & 1.000 \\
\hline Cleaning and drainage & 2 & 4 & \\
\hline Additional stenting (stent in stent) & 0 & 1 & \\
\hline Replacing stent & 12 & 6 & \\
\hline
\end{tabular}

${ }^{*} P<0.05$. ARMS: antireflux metal stent; c-CSEMS: conventional covered self-expandable metal stent.

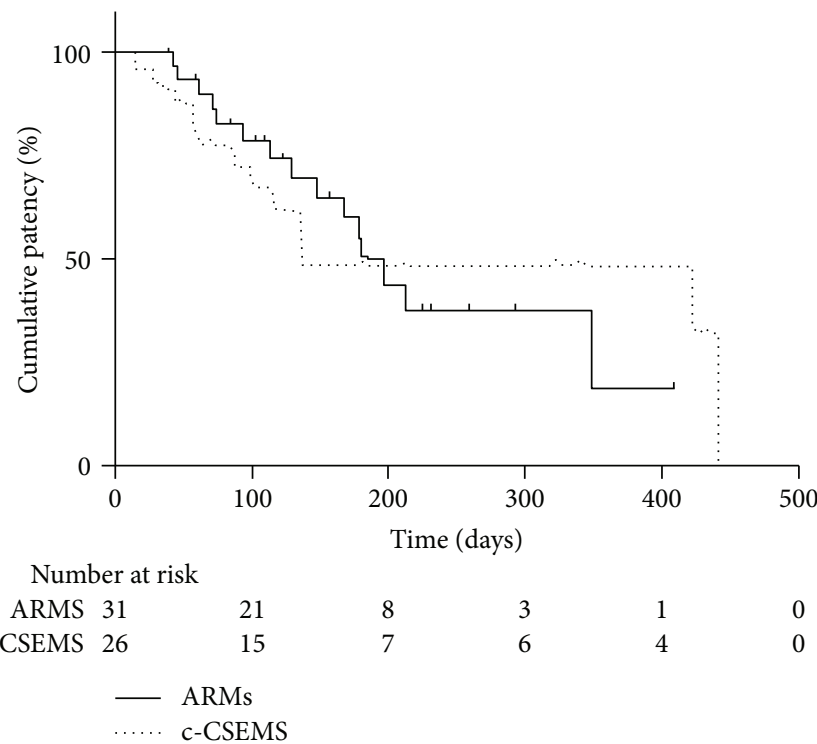

FIGURE 1: Kaplan-Meier curves showing TRBO of the ARMS and c-CSEMS groups ( $P=0.967$ by the log-rank test).

replacement ARMS. Among 3 patients with RBO due to distal migration, 2 underwent removal of the migrated stent with a conventional endoscope, and in 1 patient, the stent had already been discharged externally when RBO occurred. All of these patients received a replacement ARMS.

In the c-CSEMS group, 10 patients underwent reintervention due to $\mathrm{RBO}$. One patient improved after antibiotic administration alone. Four patients with modest sludge accumulation in the stent underwent cleaning of the stent lumen with a balloon catheter for stone removal and drainage with a nasal biliary drainage tube. In one patient, removal of the occluded stent was attempted with alligator forceps, but the stent could not be removed because of unusual resistance. In this patient, a new c-CSEMS was placed as a stent-in-stent deployment. Three patients underwent removal of the occluded stent with alligator forceps and received a new c-CSEMS. In two patients with RBO due to distal migration, the stents had already been discharged externally when RBO occurred. Both of these patients received a new c-CSEMS. In both groups, all 
reintervention procedures were performed endoscopically without any complications.

\section{Discussion}

Currently, SEMSs are used widely for unresectable MDBO, not only as palliative therapy [1-4] but also before chemotherapy $[24,25]$. They are effective for improving the quality of life in patients with a poor prognosis. In patients with MDBO, the SEMS has been proven to retain its patency for longer than a plastic stent because of its wide caliber $[8,9]$ and therefore has become standard therapy. However, this approach is not ideal for biliary obstruction, as stent occlusion may occur for various reasons such as tumor growth, sludge accumulation, food impaction, and migration. Therefore, many attempts have been made to prolong stent patency [17, 26-29].

In most patients with MDBO, the SEMS needs to be placed through the papilla. However, this results in sphincter of Oddi dysfunction, a proven cause of RBO [10, 11, 30, 31], and may cause duodenobiliary reflux whereby intestinal fluid and food residue pass through in a retrograde direction, creating a biofilm on the SEMS and sludge build-up. Additionally, the wide lumen of the SEMS may allow direct clogging by food residues. In fact, Misra and Dwivedi [11] have reported that in all patients with MDBO and placement of a SEMS, barium reflux from the duodenum to the bile duct was evident. This has prompted the development of ARMS with an antireflux valve to prevent duodenobiliary reflux. Many valve shapes are available, and their effectiveness has been shown to be comparatively good (Table 3) [12-17, 32, 33]. Hamada et al. [13] have stated that subjects with metal stents that have become occluded due to duodenobiliary reflux are good candidates for ARMS. In fact, they reported good outcomes of ARMS as a reintervention for SEMS occlusion. $\mathrm{Hu}$ et al. [16] reported a randomized controlled trial involving 112 patients treated with a nipple-shaped partially covered ARMS versus uncovered SEMS as the first-line stent for MDBO. The ARMS retained its patency significantly longer, and the frequency of cholangitis after stenting was significantly lower than that for the uncovered SEMS. In that study, however, an uncovered SEMS was employed, and this is reported to have a shorter patency duration than a covered SEMS $[26,27]$. Therefore, the basic SEMS structure might influence TRBO, and the true significance of the antireflux valve remains unclear. Lee et al. [32] developed a SEMS with a windsockshaped antireflux valve, although its basic structure was that of a classical fully covered SEMS. They reported a statistically significant improvement of TRBO with ARMS. However, since the valve length of their study was $22 \mathrm{~mm}$, there were concerns whether it could be deployed accurately via endoscopy.

In the present study, the valve of the ARMS was $7 \mathrm{~mm}$ long, and deployment was similar to that of the common SEMS. We deployed ARMS successfully in all patients initially treated for MDBO. However, as the TRBO in the ARMS group was not significantly different from that in the cCSEMS group (180.0 versus 137.0 days, $P=0.967$ ), we were unable to identify any benefit of ARMS. Univariate and multivariate analyses failed to reveal any significant risk factors for RBO, including the type of SEMS employed.

Although ARMS did prevent food impaction $(P=0.037)$, the rates of RBO due to sludge accumulation and stent migration were the same as those of c-CSEMS. There are several possible reasons for this. Although ARMS certainly prevents reflux of food residue, it cannot prevent reflux of fluids such as intestinal secretion from the duodenum and therefore formation of a biofilm on the inner membrane of the SEMS. Furthermore, formation of sludge in the valve tended to be more frequent, making the valve harder and stiffer over time, as well as shrinking the luminal space, thus leading to a sluggish and disturbed bile outflow. The antireflux valve designs with superior materials and structure have been expected, with the aim of preventing duodenobiliary reflux without interfering with antegrade bile flow. In addition, the stent migration rate in patients with $\mathrm{RBO}$ tended to be higher for ARMS than for c-CSEMS (16.1 versus 7.7\%, $P=0.436$ ). This might have been due to the nonflared stent edge of the ARMS, whereas the c-CSEMS was slightly flared at both ends. In fact, Hamada et al. [17] have reported that a newly designed ARMS with both ends flared may have a lower stent migration rate.

This is the first study to have demonstrated that, whereas the antireflux valve significantly reduces food impaction, the stent itself offers no significant advantage for initial stenting of MDBO.

Reintervention is a crucial factor affecting the prognosis of patients with MDBO. In particular, when an ARMS becomes dysfunctional, the antireflux valve has already accumulated much tough sludge. Therefore, cleaning of the stent lumen is insufficient for restoring patency, and replacement with a new stent is needed. Therefore, the possibility of SEMS removal is an important point that needs to be considered. In the present series, all patients requiring stent exchange underwent ARMS removal and replacement.

This study was limited in being retrospective and uncontrolled. Another limitation was the differences in stent structure and materials between the two groups. The basic structure of the ARMS is similar to that of the NitiS ComVi stent [21]. The type of knitting of the ComVi stent also differs from that of the Niti-S SUPREMO stent [22], corresponding to the c-CSEMS we employed. Moreover, the axial force of the ComVi stent is lower than that of the SUPREMO stent. Stents with low axial force tend to show a longer TRBO [34], and therefore the difference in axial force produced by the basic SEMS structure cannot be ignored. Furthermore, the materials of the inner luminal surfaces differed, being a silicone in the c-CSEMS and an ePTPF membrane in the ARMS. The silicone membrane is smoother than e-PTPF membrane. These differences in the inner membrane may affect the frequency of sludge accumulation or the degree of duodenobiliary reflux. Therefore, randomized controlled trials comparing ARMS with c-CSEMS with the same underlying structure and materials, except for the antireflux valve, will be needed to determine the most suitable stent for initial treatment of MDBO. 


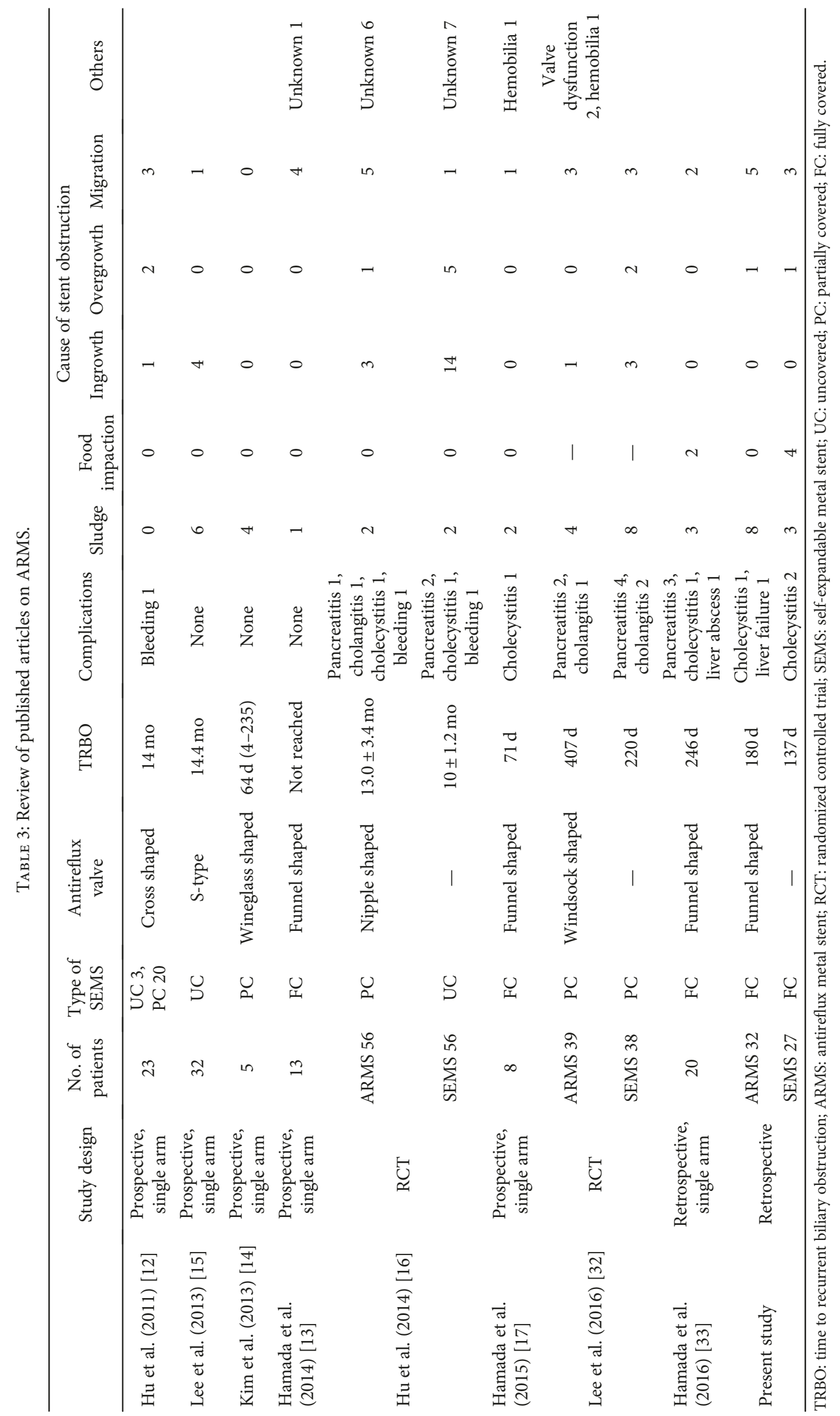




\section{Conclusions}

The present study was unable to confirm the superiority of ARMS for initial treatment of MDBO. However, RBO due to food impaction was significantly prevented by the antireflux valve. There is a possibility that the SEMS structure could be further improved or more cases suitable for use of the antireflux system identified. Further investigations are needed to evaluate the superiority of ARMS for initial treatment of MDBO.

\section{Conflicts of Interest}

The authors declare that there is no conflict of interest regarding the publication of this article.

\section{Acknowledgments}

The authors would like to thank Editage (http://www.editage .com/) for the English language editing and publication support.

\section{Supplementary Materials}

Supplementary Tables. Table S1: features of the antireflux metal stent (ARMS) and conventional covered selfexpandable metal stent (c-CSEMS). Table S2: the results of univariate and multivariate analyses for identifying $\mathrm{RBO}$ risk factors. Supplementary Figures. Figure S1: (A) antireflux covered metal stent (Niti-S long covered ComVi stent, Taewoong Medical Inc.). A funnel-shaped antireflux valve was attached at the distal end; (B) endoscopic view showing ARMS placed with its distal end in the duodenal lumen; (C) conventional covered metal stent (Niti-S SUPREMO, Taewoong Medical Inc.). The knitted structure includes large and small cells. Figure S2: (A) an ARMS was occluded because of substantial sludge sticking to its valve; (B) a vertical section of the occluded ARMS. A portion of the antireflux valve was filled with sludge (white arrowheads); (C) an ARMS (white arrows) migrated into the common bile duct (proximal migration). (Supplementary Materials)

\section{References}

[1] A. G. Speer, P. B. Cotton, R. C. Russell et al., "Randomised trial of endoscopic versus percutaneous stent insertion in malignant obstructive jaundice," Lancet, vol. 330, no. 8550, pp. 57-62, 1987.

[2] H. A. Shepherd, G. Royle, A. P. Ross, A. Diba, M. Arthur, and D. Colin-Jones, "Endoscopic biliary endoprosthesis in the palliation of malignant obstruction of the distal common bile duct: a randomized trial," British Journal of Surgery, vol. 75, no. 12 , pp. 1166-1168, 1988.

[3] J. R. Andersen, S. M. Sorensen, A. Kruse, M. Rokkjaer, and P. Matzen, "Randomised trial of endoscopic endoprosthesis versus operative bypass in malignant obstructive jaundice," Gut, vol. 30, no. 8, pp. 1132-1135, 1989.

[4] A. C. Smith, J. F. Dowsett, R. C. Russell, A. R. Hatfield, and P. B. Cotton, "Randomised trial of endoscopic steriting versus surgical bypass in malignant low bileduct obstruction," The Lancet, vol. 344, no. 8938, pp. 1655-1660, 1994.
[5] H. Yanagimoto, H. Ishii, Y. Nakai et al., "Improved survival with combined gemcitabine and S-1 for locally advanced pancreatic cancer: pooled analysis of three randomized studies," Journal of Hepato-Biliary-Pancreatic Sciences, vol. 21, no. 10, pp. 761-766, 2014.

[6] T. Conroy, F. Desseigne, M. Ychou et al., "FOLFIRINOX versus gemcitabine for metastatic pancreatic cancer," The New England Journal of Medicine, vol. 364, no. 19, pp. 1817-1825, 2011.

[7] J. Tabernero, E. G. Chiorean, J. R. Infante et al., "Prognostic factors of survival in a randomized phase III trial (MPACT) of weekly nab-paclitaxel plus gemcitabine versus gemcitabine alone in patients with metastatic pancreatic cancer," The Oncologist, vol. 20, no. 2, pp. 143-150, 2015.

[8] P. H. Davids, A. K. Groen, E. A. Rauws, G. N. Tytgat, and K. Huibregtse, "Randomised trial of self-expanding metal stents versus polyethylene stents for distal malignant biliary obstruction," The Lancet, vol. 340, no. 8834-8835, pp. 14881492, 1992.

[9] A. Schmassmann, E. von Gunten, J. Knuchel, U. Scheurer, H. F. Fehr, and F. Halter, "Wallstents versus plastic stents in malignant biliary obstruction: effects of stent patency of the first and second stent on patient compliance and survival," The American Journal of Gastroenterology, vol. 91, no. 4, pp. 654-659, 1996.

[10] T. Okamoto, S. Fujioka, S. Yanagisawa et al., "Placement of a metallic stent across the main duodenal papilla may predispose to cholangitis," Gastrointestinal Endoscopy, vol. 63, no. 6, pp. 792-796, 2006.

[11] S. P. Misra and M. Dwivedi, "Reflux of duodenal contents and cholangitis in patients undergoing self-expanding metal stent placement," Gastrointestinal Endoscopy, vol. 70, no. 2, pp. 317-321, 2009.

[12] B. Hu, T. T. Wang, Z. M. Shi et al., "A novel antireflux metal stent for the palliation of biliary malignancies: a pilot feasibility study (with video)," Gastrointestinal Endoscopy, vol. 73, no. 1, pp. 143-148, 2011.

[13] T. Hamada, H. Isayama, Y. Nakai et al., "Novel antireflux covered metal stent for recurrent occlusion of biliary metal stents: a pilot study," Digestive Endoscopy, vol. 26, no. 2, pp. 264-269, 2014.

[14] D. U. Kim, C. I. Kwon, D. H. Kang, K. H. Ko, and S. P. Hong, "New antireflux self-expandable metal stent for malignant lower biliary obstruction: in vitro and in vivo preliminary study," Digestive Endoscopy, vol. 25, no. 1, pp. 60-66, 2013.

[15] K. J. Lee, M. J. Chung, J. Y. Park et al., "Clinical advantages of a metal stent with an S-shaped anti-reflux valve in malignant biliary obstruction," Digestive Endoscopy, vol. 25, no. 3, pp. 308312, 2013.

[16] B. Hu, T. T. Wang, J. Wu, Z. M. Shi, D. J. Gao, and Y. M. Pan, "Antireflux stents to reduce the risk of cholangitis in patients with malignant biliary strictures: a randomized trial," Endoscopy, vol. 46, no. 2, pp. 120-126, 2014.

[17] T. Hamada, H. Isayama, Y. Nakai et al., “Antireflux metal stent with an antimigration system for distal malignant biliary obstruction: a feasibility pilot study," Surgical Laparoscopy Endoscopy \& Percutaneous Techniques, vol. 25, no. 3, pp. 212-217, 2015.

[18] S. I. Jang and D. K. Lee, "Stents with specialized functions: drug-eluting stents and stents with antireflux devices," Gastrointestinal Intervention, vol. 4, no. 1, pp. 50-54, 2015. 
[19] Y. Ustundag, U. Saritas, and M. Eloubeidi, "Further studies are needed on novel covered antireflux metallic stents," Endoscopy, vol. 46, no. 7, p. 627, 2014.

[20] M. M. Oken, R. H. Creech, D. C. Tormey et al., "Toxicity and response criteria of the Eastern Cooperative Oncology Group," American Journal of Clinical Oncology, vol. 5, no. 6, pp. 649656, 1982.

[21] H. Isayama, T. Kawabe, Y. Nakai et al., "Management of distal malignant biliary obstruction with the ComVi stent, a new covered metallic stent," Surgical Endoscopy, vol. 24, no. 1, pp. 131-137, 2010.

[22] T. Mukai, I. Yasuda, H. Isayama et al., "Pilot study of a novel, large-bore, fully covered self-expandable metallic stent for unresectable distal biliary malignancies," Digestive Endoscopy, vol. 28, no. 6, pp. 671-679, 2016.

[23] H. Isayama, T. Hamada, I. Yasuda et al., "TOKYO criteria 2014 for transpapillary biliary stenting," Digestive Endoscopy, vol. 27, no. 2, pp. 259-264, 2015.

[24] S. M. Wasan, W. A. Ross, G. A. Staerkel, and J. H. Lee, "Use of expandable metallic biliary stents in resectable pancreatic cancer," The American Journal of Gastroenterology, vol. 100, no. 9, pp. 2056-2061, 2005.

[25] B. R. Boulay and A. Birg, "Malignant biliary obstruction: from palliation to treatment," World Journal of Gastrointestinal Oncology, vol. 8, no. 6, pp. 498-508, 2016.

[26] A. Saleem, C. L. Leggett, M. H. Murad, and T. H. Baron, "Meta-analysis of randomized trials comparing the patency of covered and uncovered self-expandable metal stents for palliation of distal malignant bile duct obstruction," Gastrointestinal Endoscopy, vol. 74, no. 2, pp. 321-327.e3, 2011.

[27] H. Isayama, Y. Nakai, H. Kogure, N. Yamamoto, and K. Koike, "Biliary self-expandable metallic stent for unresectable malignant distal biliary obstruction: which is better: covered or uncovered?," Digestive Endoscopy, vol. 25, no. S2, pp. 71-74, 2013.

[28] H. Isayama, T. Mukai, T. Itoi et al., "Comparison of partially covered nitinol stents with partially covered stainless stents as a historical control in a multicenter study of distal malignant biliary obstruction: the WATCH study," Gastrointestinal Endoscopy, vol. 76, no. 1, pp. 84-92, 2012.

[29] M. Kitano, Y. Yamashita, K. Tanaka et al., "Covered selfexpandable metal stents with an anti-migration system improve patency duration without increased complications compared with uncovered stents for distal biliary obstruction caused by pancreatic carcinoma: a randomized multicenter trial," The American Journal of Gastroenterology, vol. 108, no. 11, pp. 1713-1722, 2013.

[30] F. Wen, Z. Lu, X. Mao, H. Liang, and Q. Guo, "Bridging across the ampulla with metal stents: evidences for intestinal bile reflux," Hepato-Gastroenterology, vol. 60, no. 128, pp. 19031905, 2013.

[31] T. Hamada, H. Isayama, Y. Nakai et al., "Duodenal invasion is a risk factor for the early dysfunction of biliary metal stents in unresectable pancreatic cancer," Gastrointestinal Endoscopy, vol. 74, no. 3, pp. 548-555, 2011.

[32] Y. N. Lee, J. H. Moon, H. J. Choi et al., "Effectiveness of a newly designed antireflux valve metal stent to reduce duodenobiliary reflux in patients with unresectable distal malignant biliary obstruction: a randomized, controlled pilot study (with videos)," Gastrointestinal Endoscopy, vol. 83, no. 2, pp. 404412, 2016
[33] T. Hamada, H. Isayama, Y. Nakai et al., “Antireflux metal stent as a first-line metal stent for distal malignant biliary obstruction: a pilot study," Gut and Liver, vol. 11, no. 1, pp. 142148, 2017.

[34] H. Isayama, Y. Nakai, Y. Toyokawa et al., "Measurement of radial and axial forces of biliary self-expandable metallic stents," Gastrointestinal Endoscopy, vol. 70, no. 1, pp. 37-44, 2009. 


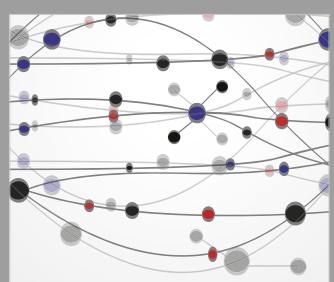

The Scientific World Journal
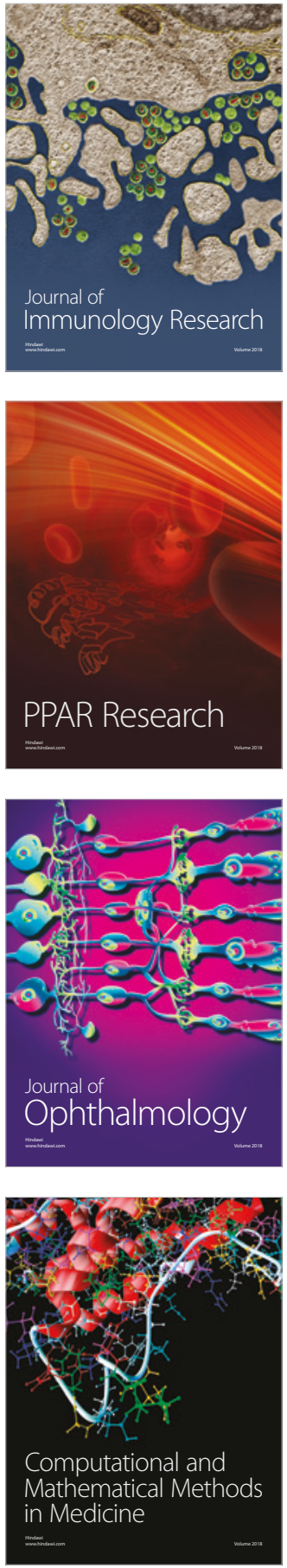

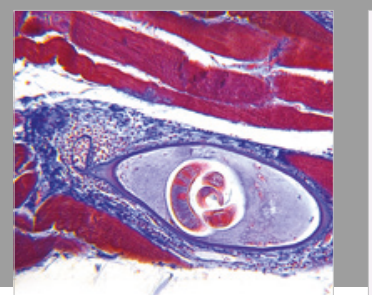

Gastroenterology Research and Practice

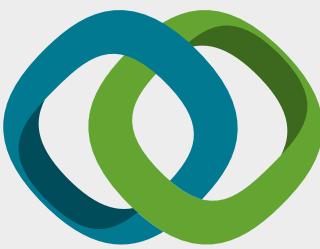

\section{Hindawi}

Submit your manuscripts at

www.hindawi.com
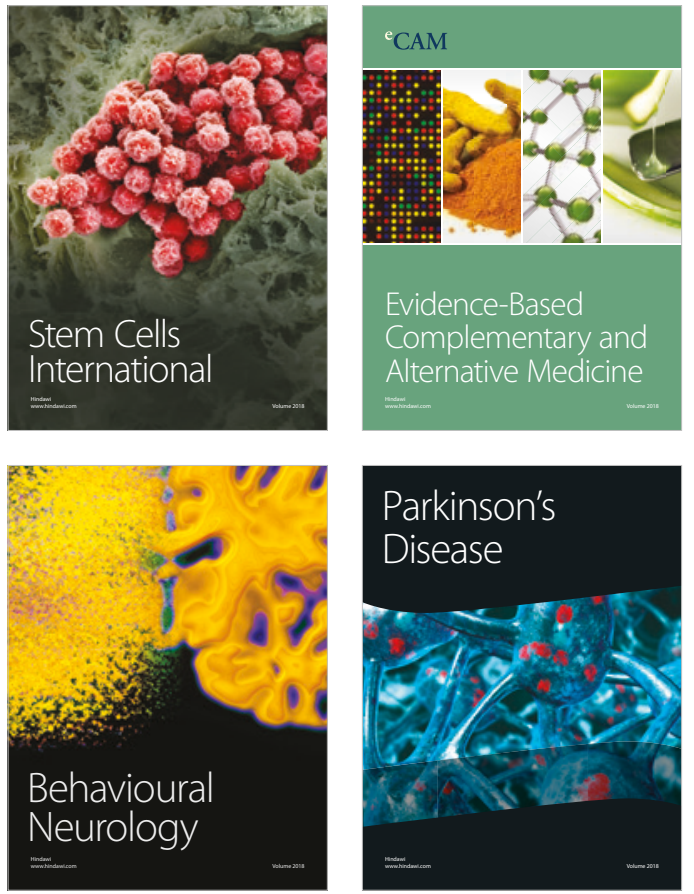

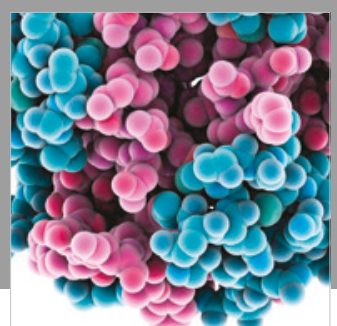

ournal of

Diabetes Research

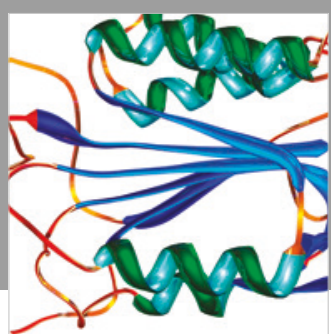

Disease Markers
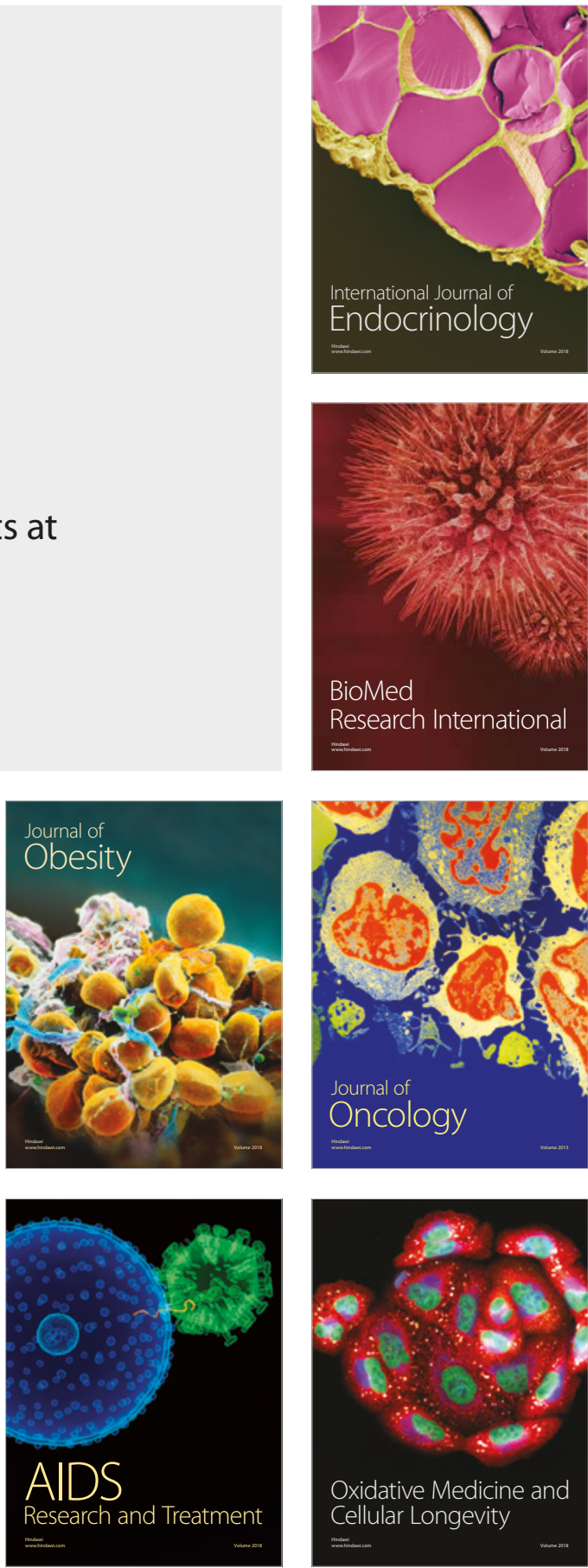